Article

\title{
Using a Microfluidic Gradient Generator to Characterize BG-11 Medium for the Growth of Cyanobacteria Synechococcus elongatus PCC7942 ${ }^{\dagger}$
}

\author{
Chih-Chun Yang ${ }^{1}$, Rex C. Wen ${ }^{2}$, Claire R. Shen ${ }^{2, *}$ and Da-Jeng Yao ${ }^{1, *}$
}

Received: 2 September 2015 ; Accepted: 5 November 2015 ; Published: 13 November 2015

Academic Editors: Wen Jung Li and Ting-Hsuan Chen

1 Institute of Nanoengineering and Microsystems, National Tsing Hua University, Hsinchu 30013, Taiwan; s102035523@m102.nthu.edu.tw

2 Department of Chemical Engineering, National Tsing Hua University, Hsinchu 30013, Taiwan; kobe810703@gmail.com

* Correspondence: crshen@mx.nthu.edu.tw (C.R.S.); djyao@mx.nthu.edu.tw (D.-J.Y.); Tel.: +886-3-5715131 (ext. 35706) (C.R.S.); +886-3-5715131 (ext. 42850) (D.-J.Y.); Fax: +886-3-5715408 (C.R.S.); +886-3-5745454 (D.-J.Y.)

$\dagger \quad$ This paper is an extended version of our paper published in the 10th Annual IEEE International Conference on Nano/Micro Engineered and Molecular Systems (IEEE NEMS 2015), Xi'an, China, 7-11 April 2015.

\begin{abstract}
The photosynthetic cyanobacterium Synechococcus elongatus PCC7942 has recently gained great attention for its ability to directly convert $\mathrm{CO}_{2}$ into renewable chemicals upon genetic engineering. Thus, it is of great interest to increase the growth speed and lower the medium requirement for cultivating this cyanobacterium. The cultivation medium of Synechococcus elongatus PCC7942 has been developed, which consists of many inorganic and metal ingredients with a specific composition, known as the BG-11 medium. In this work, we analyzed the concentration effect of each ingredient and identified the absolutely essential components in BG-11 medium for cyanobacteria growth using the concentration gradient generator chip (CGGC) fabricated by MEMS technology. As shown by our results, removal of the individual component sodium nitrate, potassium phosphate, or magnesium sulfate from the BG-11 medium led to severe growth inhibition of Synechococcus elongatus PCC7942. Contrary to our expectation, increasing concentration of the crucial ingredients showed either insignificant or negative impact on cell growth. Overall, standard growth could be achieved without supplementation of ethylenediaminetetraacetic acid (EDTA) disodium, sodium carbonate, or sodium citrate to the culture medium. Further improvement of the CGGC-based microfluidic system based on this preliminary study may broaden its application range to analyze more complicated correlations.
\end{abstract}

Keywords: cyanobacterium Synechococcus elongatus PCC7942; BG-11 medium characterization; growth optimization; microfluidic concentration gradient generator chip

\section{Introduction}

With the advance of MEMS technology, many experiments can now be conducted on the small area of chips. The application of MEMS includes, but is not limited to, Radio-Frequency (RF) MEMS [1], Optical MEMS [2], and BioMEMS [3], where micro-scale or nano-scale devices can be used as molecular sensors, mechanical actuators, or structural support. In the field of BioMEMS, the major application lies in the realm of sample detection, biochemical reaction, cell cultivation, and/or cell separation. One of the examples in medical application is the use of polydimethylsiloxane (PDMS) 
chips fabricated by MEMS to sort the mobile and immobile sperms in the micro-fluidic channel [4] to increase in vitro fertilization efficiency. The concentration gradient generator chip (CGGC) [5] is another application enabled by MEMS for the generation of a stepwise concentration gradient in high throughput experiments. Concentration gradients can be achieved automatically via two different mechanisms: static or dynamic. In the static case, concentration gradient is formed by natural diffusion without any external force. On the other hand, dynamic generation of concentration gradient relies on the help of synthetic driving forces. In recent years, extensive use of CGGC in the biological fields has been reported [6]. Generation of a stepwise concentration gradient using a micro device equipped with multiple distribution channels and confluent points was reported by Yamada et al. in 2006 [7] to examine the effect of ethanol toxicity on the growth of yeast cells. Other applications of the microfluidic concentration gradient generator ranged from analysis of the tumor and drug interactions in a dynamic hypoxia microenvironment [8] to the study of lung cancer chemotherapy resistance [9]. Design and development of an integrated radial microfluidic gradient chip was also reported for cell apoptosis assays [10]. In addition to the designs which generate a linear type of concentration gradient, several other variations including the ones that can generate non-linear or log-scale concentration profiles, have been reported [11] and applied in studies where a wide range of concentration is necessary, such as the analysis of dosage response of enzyme inhibitors. As for cyanobacteria cultivation, since cells have a physiological range of salt and mineral concentration which it can tolerate, a finer concentration scale within the biological range is preferred and therefore used in this study.

In this work, we aimed to improve the growth rate of the blue-green cyanobacteria by analyzing the essential components of the predefined growth medium using CGGC fabricated by MEMS technology. Cyanobacteria belong to a group of prokaryotic photoautotrophs that utilize light energy to fix $\mathrm{CO}_{2}$ into essential metabolites, and are collectively responsible for nearly $50 \%$ of all global photosynthetic activities [12]. Demand for a sustainable future and concern over global climate change has led to significant effort in the synthesis of commodity chemicals from renewable resources. Photosynthetic microorganisms such as cyanobacteria have therefore gained great attention for their ability to directly harvest sunlight and convert $\mathrm{CO}_{2}$ into biobased chemicals through genetic and metabolic engineering. In particular, the freshwater unicellular cyanobacterium Synechococcus elongatus PCC7942 has shown reliable transformability by exogenous DNA and has been widely engineered to synthesize various bio-monomer and biofuel molecules, such as ethylene [13], lactic acid [14], glucose [14], fructose [14], 2,3-butanediol [15], 1,2-propanediol [16], isobutyraldehyde [17], isobutanol [17], and 1-butanol [18]. Increasing growth speed of the cyanobacteria host is therefore of great interest as it would not only speed up the process of microbial engineering, but may also enhance the production efficiency of the target metabolite.

One approach to improve the growth speed of cyanobacteria in a laboratory setting is to optimize the cultivation medium and tailor the supplements according to the microorganism's need. A general cultivation medium for S. elongatus PCC7942 named BG-11 has been developed [19], and different versions $[20,21]$ based on the slight modification of this original recipe has been widely used for supporting cyanobacteria growth in the laboratory. The defined BG-11 medium consists of various inorganic salts and trace metals at a fixed concentration (Table 1). Supplementation of sodium bicarbonate to the culture medium was done at times to provide additional carbon sources in addition to the atmospheric $\mathrm{CO}_{2}$. While some of these medium ingredients are essential as the necessary nitrogen/phosphorous source, important enzyme cofactor, or buffering agent, some other components in the BG-11 medium may only be present for the purpose of generality to diversify its application. It is therefore our goal to identify the absolutely essential/non-essential ingredients for cyanobacteria growth and to characterize the medium by varying the concentration of each component in the hopes of achieving faster growth.

In contrast to the manual generation of a concentration gradient in flasks, here we employed the CGGC to automatically and systematically generate six different concentrations of each BG-11 
ingredient by setting the upper and lower concentration boundary. The goal was to create a concentration gradient from $0 \%$ to $100 \%$ with $20 \%$ interval using a microfluidic gradient generator. In this study, the concentration gradient was achieved using a Christmas tree-like microchannel structure [22], originally designed by Jeon et al. to generate and study gradient phenomenon on cell biology and surface chemistry. Similar to the CGGC design used in the testing of drug toxicity on embryonic zebrafish development reported by Yang et al. [23], our gradient chip consists of two inlets for medium introduction, which upon distribution into the Christmas tree-like microchannel, generated evenly-spaced concentration gradient among six cultivation chambers. Another similarity between our designs is the cultivation and examination of cells inside the confined chamber with manual inoculation, unlike some other reports $[24,25]$ where a continuous flow of the solution was used to cultivate cells that were attached to the chamber surface. In order for us to achieve the desired concentration distribution, flow resistance would be the most important factor. The design of long microchannel was employed in this case for optimal mixing of the fluids inside the channel. The width of the microchannel $(350 \mu \mathrm{m})$ was chosen to allow sufficient mixing since widening the channel would mean less flow resistance and therefore faster flow rates but lower mixing efficiency. Then, theoretical simulation and experimental green dye tests were performed to ensure the chip design and the flow setting were appropriate to reach the desired concentration gradient. After testing different flow rates in the system, $1.5 \mu \mathrm{L} / \mathrm{min}$ resulted in the best mixing efficiency with a concentration gradient very close to the numbers calculated by theoretical simulation. However, this was also accompanied by greater flow resistance and therefore took us a longer time for the gradient generation.

In this study, cyanobacteria growth was monitored in the microfluidic cultivation chamber with a small volume of the medium. The major results were then verified again in bench-scale flasks to ensure consistency between the microfluidic device and our regular cell cultivation vessel. As shown by our results, absence of the individual component sodium nitrate, potassium phosphate, or magnesium sulfate from the BG-11 medium caused complete growth inhibition of S. elongatus PCC7942. On the other hand, removal of ethylenediaminetetraacetic acid (EDTA) disodium, sodium carbonate, and sodium citrate had no impact on the cell growth. In contrast to our expectation, increasing concentration of the individual medium component had either an insignificant or a negative effect on the cyanobacteria growth speed. In particular, when a 10-fold higher concentration of sodium nitrate or trace metal mix was added to the medium compared to the control composition (Table 1), severe growth retardation was observed. Overall, standard growth in bench-scaled flasks could be achieved in the absence of EDTA disodium, sodium carbonate, and sodium citrate as identified by the microfluidic experiments. However, doubling the concentration of potassium phosphate, sodium nitrate, sodium bicarbonate, and/or trace metal mix failed to improve cyanobacteria growth significantly in flasks.

Table 1. Regular BG-11 medium composition used in this study as a control group. Concentration of each component was adopted from [20,21]. $50 \mathrm{mM}$ of $\mathrm{NaHCO}_{3}$ was added unless otherwise specified.

\begin{tabular}{cc}
\hline \multicolumn{2}{c}{ Final Concentration in the BG-11 Medium } \\
\hline $\mathrm{NaNO}_{3}$ & $1.5 \mathrm{~g} / \mathrm{L}$ \\
$\mathrm{CaCl}_{2}$ & $0.0272 \mathrm{~g} / \mathrm{L}$ \\
Ferric ammonium citrate & $0.012 \mathrm{~g} / \mathrm{L}$ \\
$\mathrm{Na}_{2} \mathrm{EDTA}$ & $0.001 \mathrm{~g} / \mathrm{L}$ \\
$\mathrm{K}_{2} \mathrm{HPO}_{4}$ & $0.04 \mathrm{~g} / \mathrm{L}$ \\
$\mathrm{MgSO}_{4}$ & $0.0361 \mathrm{~g} / \mathrm{L}$ \\
$\mathrm{Na}_{2} \mathrm{CO}_{3}$ & $0.02 \mathrm{~g} / \mathrm{L}$ \\
Trace minerals & $1 \mathrm{~mL} \mathrm{stock} / \mathrm{L}$ \\
Sodium citrate & $0.00882 \mathrm{~g} / \mathrm{L}$ \\
\hline
\end{tabular}

Note: * Trace mineral stock solution consists of $1.43 \mathrm{~g} \mathrm{H}_{3} \mathrm{BO}_{3}, 0.905 \mathrm{~g} \mathrm{MnCl}_{2} \cdot 4 \mathrm{H}_{2} \mathrm{O}, 0.111 \mathrm{~g} \mathrm{ZnSO}_{4} \cdot 7 \mathrm{H}_{2} \mathrm{O}$, $0.195 \mathrm{~g} \mathrm{Na}_{2} \mathrm{MoO}_{4} \cdot 2 \mathrm{H}_{2} \mathrm{O}, 0.0395 \mathrm{~g} \mathrm{CuSO}_{4} \cdot 5 \mathrm{H}_{2} \mathrm{O}, 0.0245 \mathrm{~g} \mathrm{Co}\left(\mathrm{NO}_{3}\right)_{2} \cdot 6 \mathrm{H}_{2} \mathrm{O}$ per $500 \mathrm{~mL}$ of water. 


\section{Experimental Section}

\subsection{Chemicals and Reagents}

All chemicals were purchased from Sigma-Aldrich (St. Louis, MO, USA) or JT Baker (Center Valley, PA, USA). Polydimethylsiloxane (PDMS), consisting of ingredients A and B, was purchased from Dow Corning (Midland, MI, USA).

\subsection{Device Fabrication}

The concentration gradient generator device used in this study was fabricated by polymer fabrication, which included UV lithography and PDMS molding. The detail fabrication process is shown on Figure 1a and described as follows. First, we designed the patterns for the micro-channels, which are $350 \mu \mathrm{m}$ wide with six cultivation chambers by SolidWorks (2013 x64 Edition). The acrylic matrix mold was then manufactured by a commercial company. Next, the channels were printed at 20,000 dots per inch (DPI) on transparent film (Taiwan Kong King Co. Ltd., Taoyuan, Taiwan) and used as a photomask for lithography. Then the silicon mold was fabricated in a single step under UV light using an SU8-3050 photoresist under a mask aligner. Here we obtained the bottom layer with six chambers from acrylic matrix mold. After fabricating the microfluidic device, $20 \mathrm{~g}$ of PDMS which consisted of ingredients A and B with the ratio of 10:1 was poured onto the fluidic mold placed on a petri dish to yield a fluidic layer while $55 \mathrm{~g}$ of PDMS was poured onto the acrylic matrix mold to get the base layer. After degassing by vacuum chamber, the mold was baked for $35 \mathrm{~min}$ at $80{ }^{\circ} \mathrm{C}$. Then, the PDMS layer structure was peeled from the mold. Holes were punched by a metal pin at the terminals of the inlets. Next, the fluidic layer was bonded on top of another PDMS with oxygen plasma to form the whole device. The resulting microfluidic chip is shown in Figure 1b, composed of two layers of PDMS with six cultivation chambers. Detailed fabrication parameters are as follows: spin coating speed is about $500 \mathrm{rpm} / 10 \mathrm{~s}$ followed by $1600 \mathrm{rpm} / 30 \mathrm{~s}$. The final thickness of the pattern after development is $85 \mu \mathrm{m}$ (averaged from five sites of the microchannel). The soft bake time is $95^{\circ} \mathrm{C}$ for $30 \mathrm{~min}$ and the post exposure bake (PEB) time is $65^{\circ} \mathrm{C}$ for $1 \mathrm{~min}$ followed by $95^{\circ} \mathrm{C}$ for 5 min. Plasma bonding: (1) Degas (vacuum situation) until 0.2 Torr; (2) fill oxygen until 0.5 Torr for $30 \mathrm{~s}$ to modify the PDMS surface to hydrophilic.

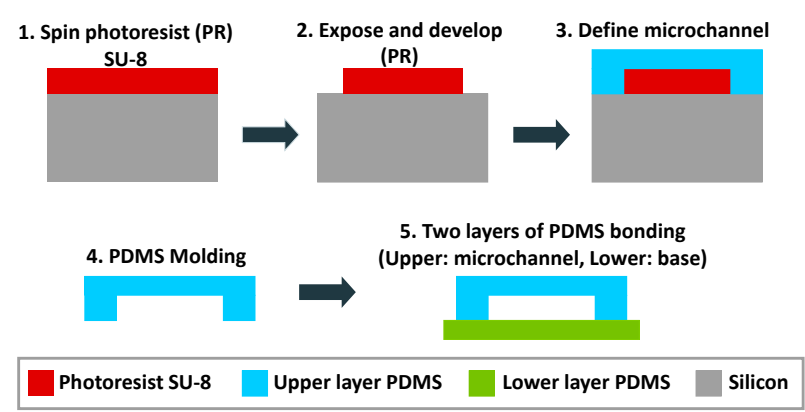

(a)

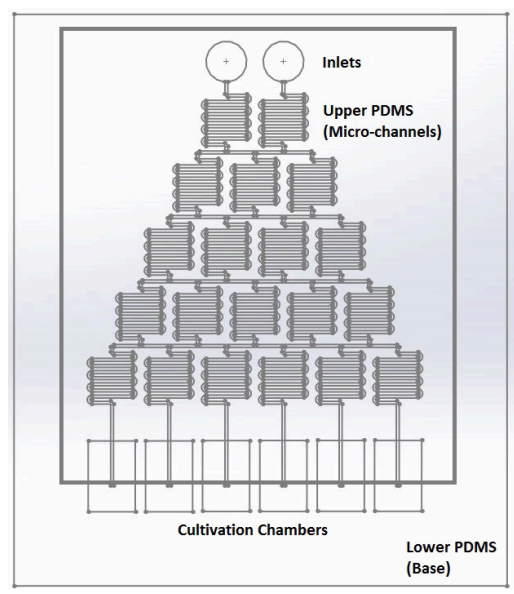

(b)

Figure 1. (a) Microfabrication process for the concentration gradient generator system; (b) Microfluidic chip composed of two layers of PDMS with six cultivation chambers.

\subsection{Design and Simulation of the Concentration Gradient Generator}

We used simulation to predict mathematical values and to estimate the concentration profile of the medium in the cultivation chamber so that a comparison between the theoretical data and 
the experimental results could be performed. The microchannel size and flow rate parameters were chosen based on prior knowledge and experiments, which generated the most stable flow distributions. The dimension of each cultivation chamber is $2 \mathrm{~cm}$ (length) $\times 0.6 \mathrm{~cm}$ (width) $\times 0.7 \mathrm{~cm}$ (height) with a total volume capacity of $840 \mu \mathrm{L}$. The full size of the chip is $9.5 \mathrm{~cm}$ (length) $\times 7 \mathrm{~cm}$ (width) $\times 0.9 \mathrm{~cm}$ (height). First, finite element analysis was conducted using CFD software (V2010.0, ESI CFD Inc., Huntsville, AL, USA) to determine the solution profile in the channels and estimate the microfluidic generation of concentration gradients. In the software CFD-GEOM, a three-dimensional model of the microfluidic channel with a width of $350 \mu \mathrm{m}$ and a height of $80 \mu \mathrm{m}$ was constructed. Therefore, the simulation result was based on a 3D structure. For the fluid field simulation, we tested three different constant flow rates $(1 \mu \mathrm{L} / \mathrm{min}, 1.5 \mu \mathrm{L} / \mathrm{min}$ and $2 \mu \mathrm{L} / \mathrm{min})$ and boundary conditions were assigned at the inlets and the walls of the channel. The simulation results were shown in Figure 2. The viscosity coefficient of green dye and BG-11 medium was measured and was shown to have the same value as deionized (DI) water. Therefore, the properties of water (density of $1000 \mathrm{~kg} / \mathrm{m}^{3}$ and viscosity of $0.7 \mathrm{~kg} \cdot \mathrm{m}^{-1} \cdot \mathrm{s}^{-1}$ ) were used to simulate the BG-11 medium in the theoretical calculations. The concentration gradient and pressure simulation showed medium distribution is well balanced and perfectly symmetrical (Figure 2). To further confirm experimentally, we ran DI water and green dye on the chip to check the mixing and concentration gradient formation. The result verified smooth medium introduction and symmetrical distribution as the green dye traveled forward with the same speed and reached the microchannel outlet at the same time (data not shown). Image J, a widely and commonly used image analyzing software, was employed to analyze the pixel ratio of the picture and then define the gradient distribution. Upon analysis, the simulation results were $100 \%, 80 \%, 60 \%, 40 \%, 20 \%$, and $0 \%$ while the experimental results (green dye test) were $100 \%, 78 \%, 59 \%, 39.5 \%, 18 \%$, and $0 \%$ under flow rate of $1.5 \mu \mathrm{L} / \mathrm{min}$.

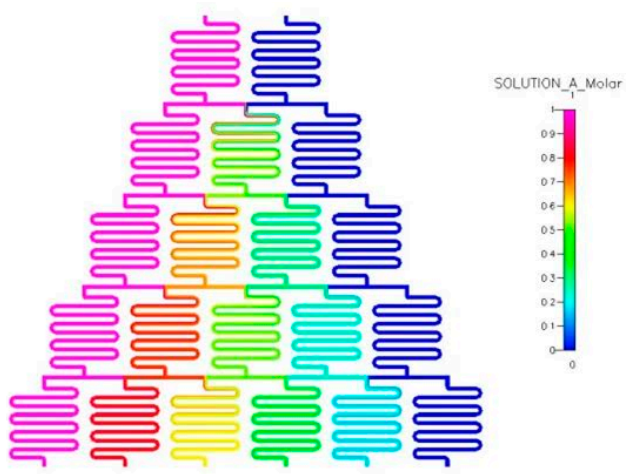

(a)

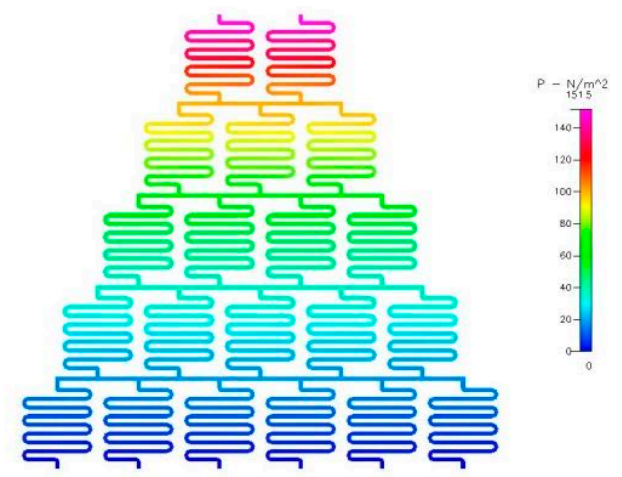

(b)

Figure 2. Simulation results by the CFD-VIEW for the analysis of concentration gradient and pressure distribution at $1.5 \mu \mathrm{L} / \mathrm{min}$ flow rate in the microchannel. (a) Concentration distribution in the microchannel with the highest concentration at the left and the lowest concentration at the right position; (b) Pressure distribution in the microchannel where pressure decreases as the solution traveled from the top to the bottom.

\subsection{Cyanobacteria Cultivation Medium and Condition}

Wild type S. elongatus PCC7942 strain was cultured in regular BG-11 medium (Table 1) containing $50 \mathrm{mM} \mathrm{NaHCO} 3$ in screwed-cap flasks or microfluidic chambers, as specified in the text. For re-streaking and maintaining cyanobacteria growth on plates, cells were grown on BG-11 agar $(1.5 \%$ $w / v)$ plates, of which the concentration of individual components is listed in Table 1. For the cases when BG-11 medium was modified with " $0 X$ " or "11.4X" of a specific component, we meant none of the specific component was added in the " $0 \mathrm{X}$ " case, or 11.4-fold higher concentration relative to the level shown on Table 1 was added in the " $11.4 X$ " case while keeping all other ingredients unchanged. The reason why we choose the number $11.4 \mathrm{X}$ was to adjust for the dilution of the specific component 
upon addition of cell resuspension into the culture medium in the cultivation chamber so that the final concentration of the specific component would be 10X (see Section 2.5 below). Cultures were grown under $35 \mu \mathrm{E} \cdot \mathrm{s}^{-1} \cdot \mathrm{m}^{-2}$ light conditions at $30^{\circ} \mathrm{C}$ with $100 \mathrm{rpm}$ shaking speed. Cell growth was monitored by measuring $\mathrm{OD}_{730}$ using the Biotek Eon spectrophotometer, in which the optical density (OD) of a cyanobacteria culture at $730 \mathrm{~nm}$ is positively correlated with the cyanobacteria cell count and is commonly used to quantify its growth.

\subsection{Generation of Concentration Gradient in the Microfluidic Chamber Using CGGC}

Before loading the culture medium into the device, we washed the micro-channels with $75 \%$ ethanol using a syringe followed by DI water twice to sterilize the channels and cultivation chambers. For each round of the experiment, we varied the concentration of one component in the BG-11 medium between " 0 " to " 10 -fold" relative to the regular BG-11 medium, except for the case of sodium bicarbonate where " 5 -fold" was set as the upper bound. To achieve the final concentration gradient of " $0,2 \mathrm{X}, 4 \mathrm{X}, 6 \mathrm{X}, 8 \mathrm{X}, 10 \mathrm{X}$ ", we manually prepared " $0 \mathrm{X}$ " and " $11.4 \mathrm{X}$ " of the specific component in BG-11 medium. After the chip pre-treatment, the modified BG-11 media with 0X and 11.4X of the specific component were loaded into the microchannel as the lower and upper boundary, and the concentration gradients were automatically generated using the syringe pump to inject the two mediums into the pre-designed CGGC, where they mixed and resulted in "0X, 2.3X, 4.6X, 6.8X, 9.1X, $11.4 X^{\prime \prime}$ of the particular ingredient. This concentration gradient was then adjusted back to the desired " $0,2 \mathrm{X}, 4 \mathrm{X}, 6 \mathrm{X}, 8 \mathrm{X}, 10 \mathrm{X}$ " upon addition of the cell resuspension $(50 \mu \mathrm{L})$ to the original medium volume of $350 \mu \mathrm{L}$ in each cultivation chamber (see Section 2.6 below). A similar concept was applied to

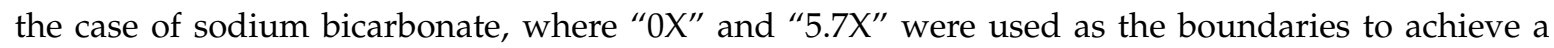
final concentration gradient of " $0 X, 1 X, 2 X, 3 X, 4 X, 5 X$ ". The injection flow rate into the CGGC was maintained constantly at $1.5 \mu \mathrm{L} / \mathrm{min}$. It took approximately $4 \mathrm{~h}$ to reach a medium volume of $350 \mu \mathrm{L}$ in each cultivation chamber. The use of bigger cultivation volume compared to other applications in the confined chamber was chosen based upon the sample loading amount requirement for measuring cell density using a spectrophotometer.

\subsection{Cyanobacteria Cultivation in the Microfluidic Chamber}

To inoculate cyanobacteria cells into the medium contained in the microfluidic chamber, we first scraped some wild type cells from the plate and resuspended them into $5 \mathrm{~mL}$ regular BG-11 medium with " $0 X^{\prime \prime}$ of the specific component (whose concentration effect is to be tested). Resuspension of cells in " $0 X$ " of the specific component avoided altering the " $0 X$ " state in the cultivation chamber upon addition of the resuspended cells into the chamber. The concentration gradient of the specific component in each chamber was prepared so that the addition of $50 \mu \mathrm{L}$ of the resuspended cells to $350 \mu \mathrm{L}$ of the modified medium would result in a final concentration gradient of $0 \mathrm{X}, 2 \mathrm{X}, 4 \mathrm{X}$, $6 \mathrm{X}, 8 \mathrm{X}$ and 10X in the cultivation chamber. To ensure all concentration tests start at the same cell density, the cell resuspension was always adjusted to an $\mathrm{OD}_{730}$ of 0.1 before inoculating $50 \mu \mathrm{L}$ into each chamber. The final volume of culture medium in each chamber was $400 \mu \mathrm{L}$ with a starting cyanobacteria cell density of around $0.01\left(\mathrm{OD}_{730}\right)$. Mixing of the cyanobacteria cell and the medium solution in the cultivation chamber was performed manually by pipetting the solution up and down during inoculation of the cells.

Once cells were inoculated into the chambers, the microfluidic chip was placed into a petri dish ( $14 \mathrm{~cm}$ diameter) and sealed with parafilm to prevent drying. The cultures were then incubated under $30{ }^{\circ} \mathrm{C}$ for $72 \mathrm{~h}$ with $100 \mathrm{rpm}$ of reciprocating motion (back-and-forth shaking). Then, cell density in each chamber was measured at $730 \mathrm{~nm}$ using a spectrophotometer.

\subsection{Verification of the Concentration Effects in Flasks}

To verify concentration effects in bench-scale flasks, different BG-11 mediums with unique concentrations of the specific component were prepared as indicated in the results section. Unless 
otherwise specified, the rest of the BG-11 constituents remained unchanged. Similar to procedures in Section 2.6 above, wild type cyanobacteria cells were scraped from the plate and resuspended into the modified BG-11 medium with an $\mathrm{OD}_{730}$ of 0.1 before inoculation. Then, $5 \mathrm{~mL}$ of the cell resuspension was added to $35 \mathrm{~mL}$ of the same modified BG-11 medium in screw-cap flasks to reach a starting cyanobacteria cell density of around $0.01\left(\mathrm{OD}_{730}\right)$. The cultures were incubated at $30{ }^{\circ} \mathrm{C}$ for a few days with $100 \mathrm{rpm}$ of orbital or reciprocating shaking. Samples were taken every day throughout the experiment by taking $200 \mu \mathrm{L}$ of the culture for cell density measurements.

\section{Results and Discussion}

\subsection{Identification of the Optimal Cultivation Volume for Cyanobacteria in the Microfluidic Chamber}

Before we could use the CGGC to test the concentration effects of various components in the BG-11 medium, we first needed to examine and identify a suitable cultivation condition for optimal cyanobacteria growth within the microfluidic chamber. Based on our design, the maximum volume allowable in the microfluidic chamber is about $840 \mu \mathrm{L}(2 \mathrm{~cm}$ in length $\times 0.6 \mathrm{~cm}$ in width $\times 0.7 \mathrm{~cm}$ in height). Since mixing of the cells by shaking the microfluidic device is necessary to avoid cell precipitation in the medium, and the extent of mixing varies with the volume loaded, we first tested cyanobacteria growth in the microfluidic chamber with $250 \mu \mathrm{L}, 300 \mu \mathrm{L}, 350 \mu \mathrm{L}, 400 \mu \mathrm{L}, 450 \mu \mathrm{L}, 500 \mu \mathrm{L}$, $550 \mu \mathrm{L}$, and $600 \mu \mathrm{L}$ of medium volume under $100 \mathrm{rpm}$ of orbital shaking. The regular BG-11 recipe (Table 1) was used as the growth medium in this test. After three days, we observed spilling and cross-contamination of cultures with a volume greater than $500 \mu \mathrm{L}$. There was also obvious cell precipitation at the bottom of each chamber across all volumes tested. We therefore hypothesized that orbital shaking may not be ideal for mixing cells contained in the rectangular-shaped cultivation chamber (Figure 1b). Switching to the mode of reciprocal shaking (back-and-forth motion) improved the extent of mixing and reduced cell precipitation significantly. Cell density of cyanobacteria reached an $\mathrm{OD}_{730}$ of about 1.0-1.2 in the chamber for medium volumes between 250 and $400 \mu \mathrm{L}$. After taking into account possible medium evaporation during the course of the experiment, we picked a working volume of $400 \mu \mathrm{L}$ for cyanobacteria cultivation in the microfluidic chamber for further experiments. We also verified that the chamber position has no impact on the cyanobacteria growth or extent of mixing by achieving similar cell density across all chamber positions with an identical medium volume (left picture in Figure 3b).

\subsection{Effects of BG-11 Medium Composition on Cyanobacteria Growth in the Microfluidic Device}

To test which BG-11 medium ingredient is absolutely essential for cyanobacteria growth and if variation of its concentration could lead to improved growth speed, we used the designed CGGC to automatically generate six different concentrations of one specific component of the BG-11 medium. A concentration gradient of " $0 \mathrm{X}, 2 \mathrm{X}, 4 \mathrm{X}, 6 \mathrm{X}, 8 \mathrm{X}, 10 \mathrm{X}$ " for a specific component relative to the level present in the regular BG-11 was generated across the microfluidic chambers, and the experiment was repeated three times for each component. The resulting cell density from each chamber after three days of incubation was then compared to the cell density obtained with regular BG-11 medium which we refer to as the "control" group. Cyanobacteria growth for the control group was repeated many times across all positions in the microfluidic chambers to ensure consistency. An average cell density of $\mathrm{OD}_{730}$ of $1.12 \pm 0.1$ was obtained as the "control" growth standard and was used as the reference to measure the impact of ingredient concentration on cell growth. We set the average "control" cell density to be $100 \%$ and displayed the results by $\%$ increase or decrease of cell growth relative to the average "control" cell density (Figure 3a). The BG-11 medium components can then be categorized into three groups based on their concentration effects on growth: essential, concentration-insensitive, and concentration-sensitive as described below. 

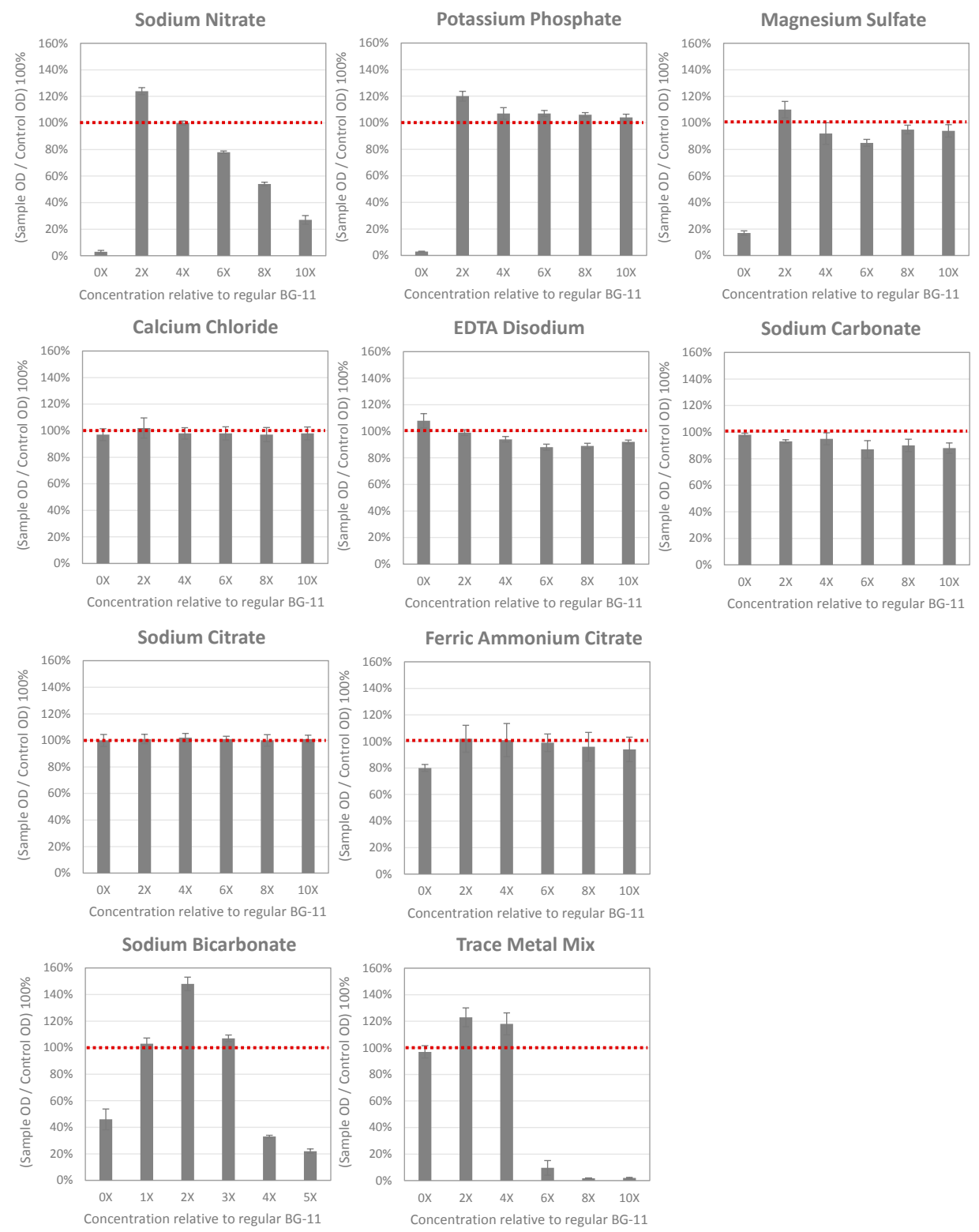

(a)

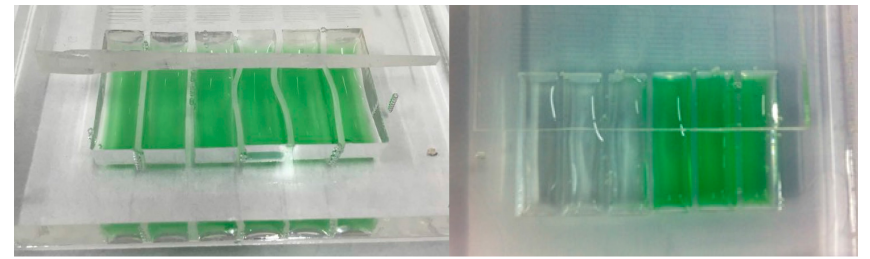

(b)

Figure 3. (a) Cyanobacteria growth comparison cultivated with different BG-11 medium composition in the microfluidic device for $72 \mathrm{~h}$. Chart title indicates the specific medium component of which the concentration was varied as shown by the $x$-axis relative to the amount used in the regular BG-11 medium (Table 1). The $y$-axis represents the $\%$ increase or decrease in cell density compared to the "control", which was cultivated under regular BG-11 medium. Standard deviation was calculated based on three repeats for the group grown under modified media. An average cell density of $\mathrm{OD}_{730}=1.12 \pm 0.1$ was obtained for the "control" group by averaging cell densities across all six cultivation chambers with many repeats; (b) Example illustration of cyanobacteria growth in the microfluidic cultivation chamber after $72 \mathrm{~h}$. Left: control group grown in regular BG-11 medium across all six chambers. Right: test group grown with different concentrations of trace metal mix in the order of $10 \mathrm{X}, 8 \mathrm{X}, 6 \mathrm{X}, 4 \mathrm{X}, 2 \mathrm{X}$, to $0 \mathrm{X}$ going from left to right. 
3.2.1. Sodium Nitrate, Potassium Phosphate, and Magnesium Sulfate are Essential Components for Cyanobacteria Growth

Cultivation of cyanobacteria in different concentrations of the individual components present in the BG-11 medium revealed the three ingredients essential for cell growth, namely sodium nitrate, potassium phosphate, and magnesium sulfate. Removal of these three ingredients individually from the medium led to complete growth inhibition in the microfluidic chamber as shown by our results. Increasing the concentration of potassium phosphate or magnesium sulfate in the medium from $2 \mathrm{X}$ to 10X relative to the regular BG-11 formula had no significant effect on the cyanobacteria growth, except that $20 \%$ higher cell density was observed in the case of $2 X$ potassium phosphate (Figure $3 a$ ). As for sodium nitrate, increasing concentration to $2 \mathrm{X}$ also showed a $20 \%$ improvement in cell growth; however, raising the concentration from $4 \mathrm{X}$ to 10X led to a proportional decrease in cell density. The level of $\mathrm{pH}$ in the modified BG-11 medium with 10X sodium nitrate was confirmed to be nearly identical ( $\mathrm{pH} \sim 8.5$ ) to the $\mathrm{pH}$ of regular BG-11. Similar observations with increasing nitrate concentration were reported in [26] where the growth of different strains of cyanobacteria was inhibited when the nitrate supplementation approached $10 \mathrm{~g} / \mathrm{L}$, a concentration much higher than those found in their natural habitat. The necessity of nitrate, phosphate, and sulfate may lie in the importance of element nitrogen, phosphorous, and sulfur in supporting the basis of life. While phosphorous is essential as the building block for the DNA backbone and the synthesis of ATP, sulfur and nitrogen are indispensable elements found in amino acids (cysteine and methionine contains sulfur) and proteins. Nitrogen also plays an important role in nucleic acid synthesis. On the other hand, magnesium participates in many enzymatic reactions as the stabilizing cofactor. Our results have verified that cyanobacteria S. elongatus PCC7942 requires the presence of a nitrogen, phosphorous, and sulfur source in the medium in order to maintain optimal growth. However, increasing the supply of these elements higher than the control recipe did not improve the growth speed more than $20 \%$.

3.2.2. Removal or Increase of Calcium Chloride, EDTA Disodium, Sodium Carbonate, or Sodium Citrate Supplementation had no Significant Effect on Cyanobacteria Growth

In contrast to the ingredients discussed in the results from Section 3.2.1 whose removal led to significant growth retardation of cyanobacteria, elimination of the following BG-11 medium components, calcium chloride, EDTA disodium, sodium carbonate, and sodium citrate, individually from the growth medium had no obvious impact on cell growth in the microfluidic chamber, except for the case of ferric ammonium citrate where a $20 \%$ decrease in cell density was observed. Elevating their concentration from $2 X$ to $10 X$ relative to the regular BG-11 did not influence the final cell density of cyanobacteria either (Figure 3a). This result may indicate the non-essentiality of these ingredients for cell growth, or for certain cases such as calcium and iron, maybe only a minute quantity possibly found in the water or chemical impurity is necessary for normal growth. Both calcium and iron are crucial elements required for the activity of signal transduction [27], electron transport in the photosystems [28], and certain classes of enzymes with Fe-S clusters. Iron is of particular importance for photosynthetic microorganisms such as cyanobacteria due to its binding to the thylakoid protein complexes for electron flow [28]. It is possible that trace amount of iron and calcium are present in our purified water or in other added chemicals so that removal of calcium chloride or ferric ammonium citrate supplementation from the cultivation medium had only a small impact on cyanobacteria growth. On the other hand, EDTA and sodium carbonate are two common ingredients found in biological buffers and growth medium. Whereas sodium carbonate may be added to form the bicarbonate buffering system, EDTA is mostly known to act as a chelating agent to scavenge metal ions when it is desired to suppress the metal ion reactivity. Citrate is a central metabolite found in the tricarboxylic acid (TCA) cycle, which for certain microorganisms may serve as a sole carbon source to support growth. In this study, we identified these five concentration-insensitive ingredients in the BG-11 medium of which variation of their concentration had no significant effect on cell growth. 
Absence of citrate, EDTA, or carbonate individually from the cultivation medium appeared to have no adverse impact on cyanobacteria growth.

3.2.3. Variation of the Trace Metal or Sodium Bicarbonate Concentration had Considerable Impact on Cyanobacteria Growth

During the course of cyanobacteria cultivation, sodium bicarbonate can be supplemented as an additional carbon source in addition to the atmospheric $\mathrm{CO}_{2}$. As shown in the Figure 3a, when the bicarbonate concentration was increased to $2 X$ that of the original level $(50 \mathrm{mM})$, cyanobacteria cell density at the 72 nd hour was improved by $40 \%$ compared to the control group. However, elevating the bicarbonate concentration more than $3 \mathrm{X}$ appeared to have a detrimental effect on cell growth, with cell density inversely proportional to the bicarbonate level. Absence of bicarbonate from the medium also led to certain degrees of growth retardation since the cells had to rely on atmospheric $\mathrm{CO}_{2}$ as the sole carbon source. Trace metal is another group of ingredients which had a significant impact on cell growth as its concentration was varied. The trace metal mixture consists of six different metal compounds, including $\mathrm{H}_{3} \mathrm{BO}_{3}, \mathrm{MnCl}_{2}, \mathrm{ZnSO}_{4}, \mathrm{Na}_{2} \mathrm{MoO}_{4}, \mathrm{CuSO}_{4}$, and $\mathrm{Co}\left(\mathrm{NO}_{3}\right)_{2}$. As we know, trace metals play an essential role in mediating the basic cellular metabolism and enzymatic reactions. In particular, certain metal ions such as $\mathrm{Zn}, \mathrm{Mn}$, and $\mathrm{Cu}$ may have indispensable function in photosynthesis and carbon fixation as they are essential enzyme cofactors involved in water oxidation, electron transfer, and $\mathrm{CO}_{2}$ assimilation [28]. Nevertheless, it is possible that very low amounts of such metals are required by cyanobacteria to maintain normal cellular function. As a result, if trace amount had been present in the medium from water or chemical impurity, it would explain why removal of the trace metal mixture entirely from the cultivation medium had no apparent effect on cell growth. We observed a $20 \%$ increase in cell density when the trace metal concentration was raised to $2 \mathrm{X}$ relative to the control level. Interestingly, when the trace metal concentration surpassed 6X in the medium, complete growth inhibition of cyanobacteria was observed (Figure 3a, b-right). It is possible that an excessive concentration of the trace metals has inhibitory effects on essential enzymes and/or cellular regulations. The level of $\mathrm{pH}$ in the modified BG-11 medium with 10X sodium bicarbonate or 10X trace metal mix was confirmed to be nearly identical ( $\mathrm{pH} \sim 8.5$ ) to the $\mathrm{pH}$ of regular BG-11.

\subsection{Verification of Medium Composition Effects on Growth in Bench-Scale Flasks}

To verify that the concentration effects observed in the microfluidic chamber is not a vessel-dependent observation and similar effects would also be reflected in bench-scale flasks, we conducted growth experiments of cyanobacteria in screwed-cap flasks with medium compositions that showed significant growth differences from the microfluidic tests. The resulting growth trend over the course of four days is then compared to the one obtained from the control group, which was grown under the regular BG-11 medium recipe (Table 1). As shown in Figure 4, elimination of sodium nitrate, potassium phosphate, or magnesium sulfate supplementation individually from the cultivation medium resulted in similar growth inhibition in flasks as observed in the microfluidic chamber experiments, confirming their necessity for cyanobacteria growth. On the other hand, individual removal of calcium chloride, EDTA disodium, sodium carbonate, sodium citrate, or the trace metal mix from the medium did not cause much adverse effect on growth in the flasks throughout the course of cultivation. Cell growth appeared to be completely unharmed by the elimination of EDTA disodium, sodium carbonate, and sodium citrate supplementation from the culture medium. We also verified that removal of the aforementioned five ingredients simultaneously from the medium was not significantly detrimental as seen from Figure 4. Absence of ferric ammonium citrate supplementation was a bit more harmful to growth when cultivated in flasks than in the microfluidic device, where more than a $50 \%$ decrease in cell density was observed in flasks. Finally, we tested if similar $20 \%-40 \%$ growth enhancement of cyanobacteria as shown by the microfluidic experiments can be achieved in flasks with the addition of $2 \mathrm{X}$ potassium phosphate, $2 \mathrm{X}$ 
sodium nitrate, $2 \mathrm{X}$ sodium bicarbonate, and/or $2 \mathrm{X}$ trace metal mix. Parallel to the results obtained from the microfluidic chip, doubling the concentration of these four components did consistently increase cyanobacteria growth by $10 \%-20 \%$ compared to the control group; however, the growth improvement was not very significant when taking into account the growth deviation in different batches of the control experiment.
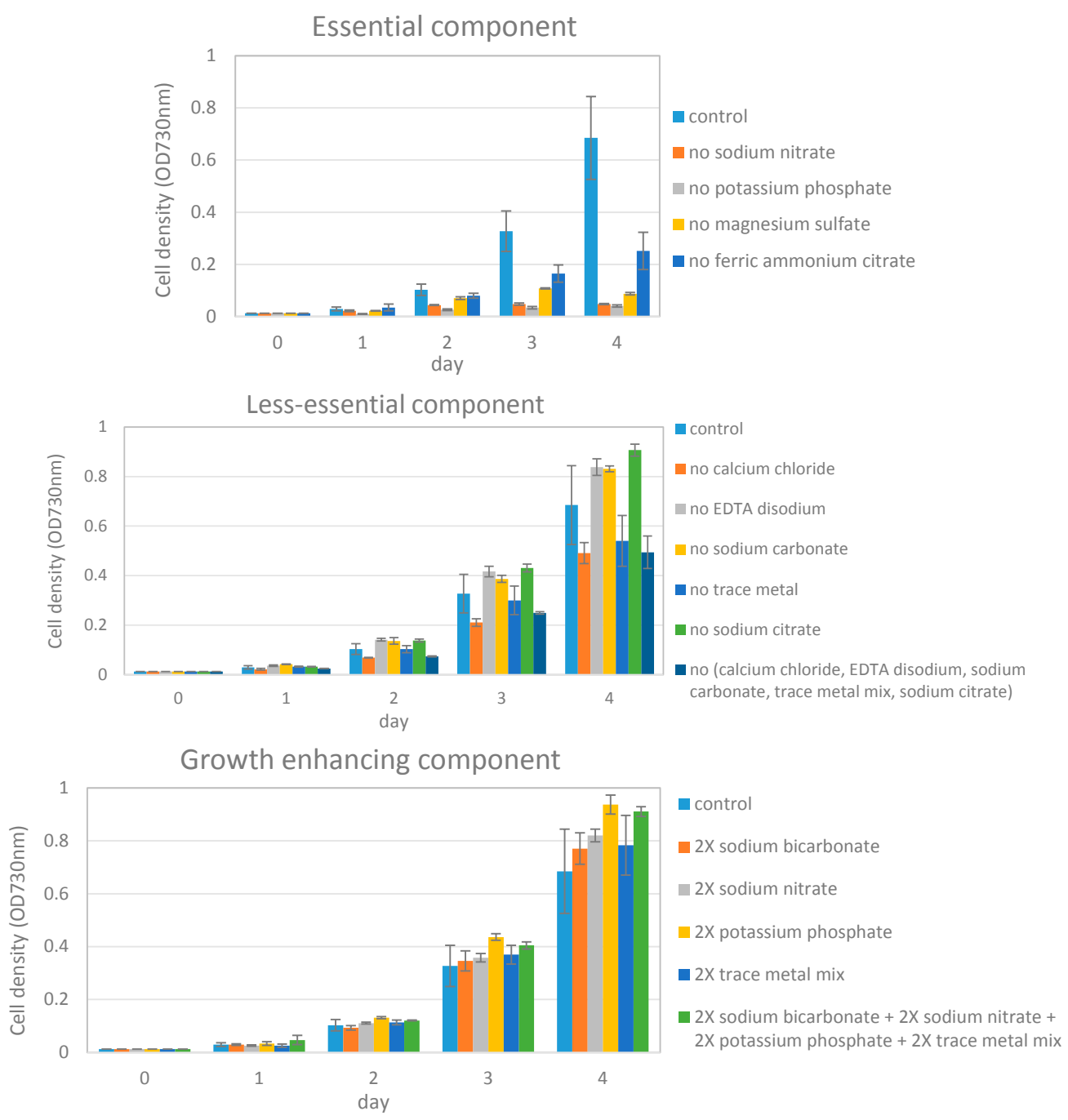

Figure 4. Verification of significant findings from the microfluidic experiments in bench-scale flasks. The results were grouped into three categories, essential, less essential, and growth enhancing depending on if their supplementation was necessary to maintain normal cell growth in flasks compared to the control (grown under regular BG-11 medium). For all the tests shown here, the BG-11 medium was modified according to the figure legends shown.

\section{Conclusions}

With the CGGC fabricated by the MEMS technology, we were able to build a concentration gradient automatically and use it for many different applications. In this study, analysis of the BG-11 medium composition on cyanobacteria growth was performed by generating concentration gradients of the individual medium components using CGGC. The ultimate goal of our work is to examine the complicated relationship between multi- concentration gradients and cell growth with variation of more than one medium component at the same time, as realized in 2D systems $[29,30]$. This study presents a preliminary analysis of the accuracy of a microfluidic chip-based generation of concentration gradients and cyanobacteria cultivation. The results shown here demonstrated high 
consistency between CGGC-based growth response and bench-scale growth behavior with different medium composition, which solidifies the foundation for future design and analysis of complex medium and growth relationship using a 2D system.

Upon optimization of the working volume in the microfluidic chamber for cyanobacteria cultivation, we identified the absolutely essential and potentially non-essential ingredients in the BG-11 medium. Sodium nitrate, potassium phosphate, and magnesium sulfate are the three important components that had to be supplemented for normal cell growth; however, increasing their concentration did not result in better growth speed. Apart from the case of ferric ammonium citrate where its removal led to obvious growth retardation, the rest of the BG-11 medium components, including calcium chloride, EDTA disodium, sodium carbonate, sodium citrate, and the trace metal mix, appear to be non-essential or required in such trace amounts that removal of their supplementation did not cause significant harm to cell growth. Among them, sodium nitrate, sodium bicarbonate, and the trace metal mix are the ingredients that would hurt growth if their concentrations were raised above $4 X$ relative to the regular BG- 11 recipe. The rest of the components had no effect on cell growth with increasing concentrations up to 10X. Further expansion of the CGGC design to a 2D system will potentially allow us to analyze and discover more complex correlations between growth and medium composition.

Acknowledgments: This work was supported by the Ministry of Science and Technology with grant \#103-2218-E-007-009-MY2.

Author Contributions: Chih-Chun Yang designed and performed the experiment in the microfluidic chip, acquired and analyzed the data, and drafted the manuscript. Rex C. Wen performed the experiment in flasks, acquired and analyzed the data. Claire R. Shen designed and supervised the experiment concerning cyanobacteria cultivation, analyzed the data and wrote the manuscript. Da-Jeng Yao designed and supervised the experiment concerning the microfluidic device and proofread the manuscript.

Conflicts of Interest: The authors declare no conflict of interest.

\section{References}

1. Huff, G.H.; Bernhard, J.T. Integration of packaged RF MEMS switches with radiation pattern reconfigurable square spiral microstrip antennas. IEEE Trans. Antennas Propag. 2006, 54, 464-469. [CrossRef]

2. Solgaard, O.; Godil, A.A.; Howe, R.T.; Lee, L.P.; Peter, Y.A.; Zappe, H. Optical MEMS: From Micromirrors to Complex Systems. J. Microelectromech. Syst. 2014, 23, 517-538. [CrossRef]

3. Jivani, R.R.; Lakhtaria, G.J.; Patadiya, D.D.; Patel, L.D.; Jivani, N.P.; Jhala, B.P. Biomedical microelectromechanical systems (BioMEMS): Revolution in drug delivery and analytical techniques. Saudi Pharm. J. 2013. [CrossRef]

4. Huang, H.-Y.; Huang, P.-W.; Yao, D.-J. Enhanced efficiency of sorting sperm motility utilizing a microfluidic chip. Microsyst. Technol. 2015. [CrossRef]

5. Tirella, A.; Marano, M.; Vozzi, F.; Ahluwalia, A. A microfluidic gradient maker for toxicity testing of bupivacaine and lidocaine. Toxicol. Vitro 2008, 22, 1957-1964. [CrossRef] [PubMed]

6. Kim, S.; Kim, H.J.; Jeon, N.L. Biological applications of microfluidic gradient devices. Integr. Biol. 2010, 2, 584-603. [CrossRef] [PubMed]

7. Yamada, M.; Hirano, T.; Yasuda, M.; Seki, M. A microfluidic flow distributor generating stepwise concentrations for high-throughput biochemical processing. Lab Chip 2006, 6, 179-184. [CrossRef] [PubMed]

8. Wang, L.; Liu, W.; Wang, Y.; Wang, J.-C.; Tu, Q.; Liu, R.; Wang, J. Construction of oxygen and chemical concentration gradients in a single microfluidic device for studying tumor cell-drug interactions in a dynamic hypoxia microenvironment. Lab Chip 2013, 13, 695-705. [CrossRef] [PubMed]

9. Wang, S.Y.; Yue, F.; Zhang, L.C.; Wang, J.R.; Wang, Y.Y.; Jiang, L.; Lin, B.C.; Wang, Q. Application of microfluidic gradient chip in the analysis of lung cancer chemotherapy resistance. J. Pharm. Biomed. Anal. 2009, 49, 806-810.

10. Yang, C.G.; Wu, Y.F.; Xu, Z.R.; Wang, J.H. A radial microfluidic concentration gradient generator with high-density channels for cell apoptosis assay. Lab Chip 2011, 11, 3305-3312. [CrossRef] [PubMed] 
11. Yun, J.Y.; Jambovane, S.; Kim, S.K.; Cho, S.H.; Duin, E.C.; Hong, J.W. Log-Scale Dose Response of Inhibitors on a Chip. Anal. Chem. 2011, 83, 6148-6153. [CrossRef] [PubMed]

12. Field, C.B.; Behrenfeld, M.J.; Randerson, J.T.; Falkowski, P. Primary production of the biosphere: Integrating terrestrial and oceanic components. Science 1998, 281, 237-240. [CrossRef] [PubMed]

13. Ungerer, J.; Tao, L.; Davis, M.; Ghirardi, M.; Maness, P.C.; Yu, J.P. Sustained photosynthetic conversion of $\mathrm{CO}_{2}$ to ethylene in recombinant cyanobacterium Synechocystis 6803. Energy Environ. Sci. 2012, 5, 8998-9006. [CrossRef]

14. Niederholtmeyer, H.; Wolfstadter, B.T.; Savage, D.F.; Silver, P.A.; Way, J.C. Engineering Cyanobacteria to Synthesize and Export Hydrophilic Products. Appl. Environ. Microbiol. 2010, 76, 3462-3466. [CrossRef] [PubMed]

15. Oliver, J.W.K.; Machado, I.M.P.; Yoneda, H.; Atsumi, S. Cyanobacterial conversion of carbon dioxide to 2,3-butanediol. Proc. Natl. Acad. Sci. USA 2013, 110, 1249-1254. [CrossRef] [PubMed]

16. Li, H.; Liao, J.C. Engineering a cyanobacterium as the catalyst for the photosynthetic conversion of $\mathrm{CO}_{2}$ to 1,2-propanediol. Microb. Cell Fact. 2013, 12, 4. [CrossRef] [PubMed]

17. Atsumi, S.; Higashide, W.; Liao, J.C. Direct photosynthetic recycling of carbon dioxide to isobutyraldehyde. Nat. Biotechnol. 2009, 27, 1177-1180. [CrossRef] [PubMed]

18. Lan, E.I.; Liao, J.C. ATP drives direct photosynthetic production of 1-butanol in cyanobacteria. Proc. Natl. Acad. Sci. USA 2012, 109, 6018-6023. [CrossRef] [PubMed]

19. Allen, M.M. Simple conditions for growth of unicellular blue-green algae on plates. J. Phycol. 1968, 4, 1-4. [CrossRef]

20. Bustos, S.A.; Golden, S.S. Expression of the Psbdii Gene in Synechococcus sp. Strain PCC 7942 Requires Sequences Downstream of the Transcription Start Site. J. Bacteriol. 1991, 173, 7525-7533. [PubMed]

21. Stanier, R.Y.; Kunisawa, R.; Mandel, M.; Cohen-Bazire, G. Purification and properties of unicellular blue-green algae (order Chroococcales). Bacteriol. Rev. 1971, 35, 171-205. [PubMed]

22. Jeon, N.L.; Dertinger, S.K.W.; Chiu, D.T.; Choi, I.S.; Stroock, A.D.; Whitesides, G.M. Generation of solution and surface gradients using microfluidic systems. Langmuir 2000, 16, 8311-8316. [CrossRef]

23. Yang, F.; Chen, Z.G.; Pan, J.B.; Li, X.C.; Feng, J.; Yang, H. An integrated microfluidic array system for evaluating toxicity and teratogenicity of drugs on embryonic zebrafish developmental dynamics. Biomicrofluidics 2011, 5, 24115. [CrossRef] [PubMed]

24. Choudhury, D.; van Noort, D.; Iliescu, C.; Zheng, B.X.; Poon, K.L.; Korzh, S.; Korzh, V.; Yu, H. Fish and Chips: A microfluidic perfusion platform for monitoring zebrafish development. Lab Chip 2012, 12, 892-900. [CrossRef] [PubMed]

25. Hsiung, L.C.; Chiang, C.L.; Wang, C.H.; Huang, Y.H.; Kuo, C.T.; Cheng, J.Y.; Lin, C.H.; Wu, V.; Chou, H.Y.; Jong, D.S.; et al. Dielectrophoresis-based cellular microarray chip for anticancer drug screening in perfusion microenvironments. Lab Chip 2011, 11, 2333-2342. [CrossRef] [PubMed]

26. Ernst, A.; Deicher, M.; Herman, P.M.J.; Wollenzien, U.I.A. Nitrate and phosphate affect cultivability of cyanobacteria from environments with low nutrient levels. Appl. Environ. Microbiol. 2005, 71, 3379-3383. [CrossRef] [PubMed]

27. Shi, J.Q.; Wu, Z.X.; Song, L.R. Physiological and molecular responses to calcium supplementation in Microcystis aeruginosa (Cyanobacteria). N. Z. J. Mar. Freshw. Res. 2013, 47, 51-61. [CrossRef]

28. Raven, J.A.; Evans, M.C.W.; Korb, R.E. The role of trace metals in photosynthetic electron transport in $\mathrm{O}_{2}$-evolving organisms. Photosynth. Res. 1999, 60, 111-149. [CrossRef]

29. Hu, S.W.; Xu, B.Y.; Xu, J.J.; Chen, H.Y. Liquid gradient in two-dimensional matrix for high throughput screening. Biomicrofluidics 2013, 7, 64116. [CrossRef] [PubMed]

30. Jang, Y.H.; Hancock, M.J.; Kim, S.B.; Selimovic, S.; Sim, W.Y.; Bae, H.; Khademhosseini, A. An integrated microfluidic device for two-dimensional combinatorial dilution. Lab Chip 2011, 11, 3277-3286. [CrossRef] [PubMed]

(C) 2015 by the authors; licensee MDPI, Basel, Switzerland. This article is an open access article distributed under the terms and conditions of the Creative Commons by Attribution (CC-BY) license (http:/ / creativecommons.org/licenses/by/4.0/). 